\title{
Opportunistic Information Dissemination in Mobile Ad-hoc Networks: The Profit of Global Synchrony *
}

\author{
Antonio Fernández Anta ${ }^{1,2}$, Alessia Milani ${ }^{3}$, \\ Miguel A. Mosteiro ${ }^{4,2}$, and Shmuel Zaks ${ }^{5}$ \\ ${ }^{1}$ Institute IMDEA Networks, Leganés, Spain \\ 2 LADyR, GSyC, Universidad Rey Juan Carlos, Móstoles, Spain \\ anto@gsyc.es \\ 3 LIP6, Université Pierre et Marie Curie - Paris 6, Paris, France \\ alessia.milani@lip6.fr \\ ${ }^{4}$ Department of Computer Science, Rutgers University, Piscataway, NJ, USA \\ mosteirodcs.rutgers.edu \\ ${ }^{5}$ Department of Computer Science, Technion, Haifa, Israel \\ zaks@cs.technion.ac.il
}

\begin{abstract}
The topic of this paper is the study of Information Dissemination in Mobile Ad-hoc Networks by means of deterministic protocols. We characterize the connectivity resulting from the movement, from failures and from the fact that nodes may join the computation at different times with two values, $\alpha$ and $\beta$, so that, within $\alpha$ time slots, some node that has the information must be connected to some node without it for at least $\beta$ time slots. The protocols studied are classified into three classes: oblivious (the transmission schedule of a node is only a function of its ID), quasi-oblivious (the transmission schedule may also depend on a global time), and adaptive.

The main contribution of this work concerns negative results. Contrasting the lower and upper bounds derived, interesting complexity gaps among protocolclasses are observed. More precisely, in order to guarantee any progress towards solving the problem, it is shown that $\beta$ must be at least $n-1$ in general, but that $\beta \in \Omega\left(n^{2} / \log n\right)$ if an oblivious protocol is used. Since quasi-oblivious protocols can guarantee progress with $\beta \in O(n)$, this represents a significant gap, almost linear in $\beta$, between oblivious and quasi-oblivious protocols. Regarding the time to complete the dissemination, a lower bound of $\Omega\left(n \alpha+n^{3} / \log n\right)$ is proved for oblivious protocols, which is tight up to a polylogarithmic factor because a constructive $O\left(n \alpha+n^{3} \log n\right)$ upper bound exists for the same class. It is also proved that adaptive protocols require $\Omega\left(n \alpha+n^{2}\right)$, which is optimal given that a matching upper bound can be proved for quasi-oblivious protocols. These results show that the gap in time complexity between oblivious and quasioblivious, and hence adaptive, protocols is almost linear. This gap is what we call the profit of global synchrony, since it represents the gain the network obtains from global synchrony with respect to not having it.
\end{abstract}

\footnotetext{
* This research was partially supported by Spanish MICINN grant no. TIN2008-06735-C02-01, Comunidad de Madrid grant no. S2009TIC-1692, EU Marie Curie International Reintegration Grant IRG 210021, NSF grant no. 0937829, ANR grant R-DISCOVER, and by the Israel Science Foundation, grant no. 1249/08
} 


\section{Introduction}

A Mobile Ad-hoc Network (aka MANET) is a set of mobile nodes which communicate over a multihop radio network, without relying on a stable infrastructure. In these networks, nodes are usually battery-operated devices that can communicate via radio with other devices that are in range. Due to unreliable power supply and mobility, nodes may have a continuously changing set of neighbors in that range. This dynamic nature makes it challenging to solve even the simplest communication problems in general. Hence, proposed protocols often have strong synchronization and stability requirements, like having a stable connected network for long enough time.

Current trends in networking-architecture developments, like delay and disruption tolerant networks, and opportunistic networking [8,22], aim to deal with the disconnections that naturally and frequently arise in wireless environments. Their objective is to allow communication in dynamic networks, like a MANET, even if a route between sender and receiver never exists in the network. The result is that multi-hop communication is provided through opportunistic communication, in which the online route of a message is followed one link at a time, as links in the route become available. While the next link is not available, the message is held in a node. With opportunistic communication, strong connectivity requirements are no longer needed. Furthermore, in some cases mobility is the key to allow communication (e.g., consider two disconnected static nodes, where communication between them is provided by a device that, due to mobility, sometimes is in range of one and sometimes of the other).

In this paper, we formally define a particular class of MANET which is suited for opportunistic communication, and which we call potentially epidemic. A MANET is potentially epidemic if the changes in the communication topology are such that an online route exists among any two nodes that wish to communicate.

The network is potentially epidemic because the actual propagation of the information on the online routes, and then the possibility for a node to affect another node, depends on the stability of each communication links of the online route.

In this context, we define and study the deterministic solvability of a problem that we call Dissemination. In this problem, at a given time a source node holds an information that must be disseminated to a given set of nodes belonging to the MANET. The nodes elected to eventually receive the information are the ones that satisfy a given predicate. Depending on this predicate, the Dissemination problem can instantiate most of the common communication problems in distributed systems, such as Broadcast, Multicast, Geocast, Routing, etc.

In particular, we determine assumptions on link stability and speed of nodes under which a distributed deterministic protocol exists that solves Dissemination in potentially epidemic networks. Moreover, we relate the time complexity of the solution to the speed of movement and to the information that protocols may use.

\subsection{The Dissemination Problem}

We study the problem of disseminating a piece of information, initially held by a distinguished source node, to all nodes of a given set in the network. Formally, 
Definition 1. Given a MANET formed by a set $V$ of $n$ nodes, let $\mathcal{P}$ be a predicate on $V$ and $s \in V$ a node that holds a piece of information $I$ at time $t_{1}$ ( $s$ is the source of dissemination). The Dissemination problem consists of distributing I to the set of nodes $V_{\mathcal{P}}=\{x \in V:: \mathcal{P}(x)\}$. A node that has received $I$ is termed covered, and otherwise it is uncovered. The Dissemination problem is solved at time slot $t_{2} \geq t_{1}$ if, for every node $v \in V_{\mathcal{P}}$, $v$ is covered by time slot $t_{2}$.

The Dissemination problem abstracts several common problems in distributed systems. E.g. Broadcast, Multicast, Geocast, Routing etc., are all instances of this problem for a particular predicate $\mathcal{P}$. In order to prove lower bounds, we will use one of these instances: the Geocast problem. The predicate $\mathcal{P}$ for Geocast is $\mathcal{P}(x)=$ true if and only if, at time $t_{1}, x$ is up and running, and it is located within a parametric distance $d>0$ (called eccentricity) from the position of the source node at that time.

\subsection{Model}

We consider a MANET formed by a set $V$ of $n$ mobile nodes deployed in $\mathbb{R}^{2}$, where no pair of nodes can occupy the same point in the plane simultaneously. It is assumed that each node has data-processing and radio-communication capabilities, and a unique identificator number (ID) in $[n] \triangleq\{1, \ldots, n\}$.

Time. Each node is equipped with a clock that ticks at the same uniform rate $\rho$ but, given the asynchronous activation, the clocks of different nodes may start at different times. A time interval of duration $1 / \rho$ is long enough to transmit (resp. receive) a message. Computations in each node are assumed to take no time. Starting from a time instant used as reference, the global time is slotted as a sequence of time intervals or time slots $1,2, \ldots$, where slot $i>0$ corresponds to the time interval $[(i-1) / \rho, i / \rho)$. Without loss of generality [24] all node's ticks are assumed to be in phase with this global tick.

Node Activation. We say that a node is active if it is powered up, and inactive otherwise. It is assumed that, due to lack of power supply or other unwanted events that we call failures, active nodes may become inactive. Likewise, due also to arbitrary events such as replenishing their batteries, nodes may be re-activated. We call the temporal sequence of activation and failures of a node the activation schedule. The activation schedule for each node is assumed to be chosen by an adversary, in order to obtain worst-case bounds. Most of the lower-bound arguments included in this paper hold, even if all nodes are activated simultaneously and never fail (which readily provide a global time), making the results obtained stronger.

We assume that a node is activated in the boundary between two consecutive time slots. If a node is activated between slots $t-1$ and $t$ we say that it is activated at slot $t$, and it is active in that slot. Upon activation, a node immediately starts running from scratch an algorithm previously stored in its hardware, but no other information or status is preserved while a node is inactive. Consequently, it is possible that a covered node does not hold the information $I$, because it has been inactive after receiving it. To distinguish a covered node that does not hold the information from one that holds it, we introduce the following additional terminology: we say that a node $p$ is informed at a given time $t$ if it holds the information $I$ at time $t$, otherwise $p$ is said to be uninformed. 
Radio Communication. Nodes communicate via a collision-prone single radio channel. A node $v$ can receive a transmission of another node $u$ in time slot $t$ only if their distance is at most the range of transmission $r$ during the whole slot $t$. The range of transmission is assumed to be the same for all nodes and all time slots. If two nodes $u$ and $v$ are separated by a distance at most $r$, we say that they are neighbors. In this paper, no collision detection mechanism is assumed, and a node cannot receive and transmit at the same time slot. Therefore, an active node $u$ receives a transmission from a neighboring node $v$ at time slot $j$ if and only if $v$ is the only node in $u$ 's neighborhood transmitting at time slot $j$. Also, a node cannot distinguish between a collision and no transmission. In general, we say that a node $v \in V^{\prime}$ transmits uniquely among the nodes of set $V^{\prime} \subseteq V$ in a slot $t$ if it is the only node in $V^{\prime}$ that transmits in $t$.

Link stability. We assume that nodes may move on the plane. Thus, the topology of the network is time dependent. For simplicity, we assume that the topology only changes in the boundaries between time slots. Then, at time slot $t$ nodes $u$ and $v$ are connected by a link in the network topology iff they are neighbors during the whole slot $t$. An online route between two nodes $u$ and $v$ is a path $u=w_{0}, w_{1}, \ldots, w_{k}=v$ and a sequence of time slots $t_{1}<t_{2}<\cdots<t_{k}$ such that the network has a link between $w_{i-1}$ and $w_{i}$ at time slot $t_{i}$. Observe that in order to be able to solve an instance of Dissemination, it is necessary that the network is potentially epidemic. I.e. after the initial time $t_{1}$, there is an online route from the source $s$ to every node in $V_{\mathcal{P}}$. However, as argued in [6], worst-case adversarial choice of topologies for a dynamic network precludes any deterministic protocol from completing Broadcast, even if connectivity is guaranteed. Note that Broadcast is an instance of Dissemination, and that if there is connectivity then there are online routes between all nodes. Thus, the property that the network is potentially epidemic as described is not sufficient to solve Dissemination, and further limitations to the adversarial movement and activation schedule are in order. While respecting a bound on the maximum speed $v_{\max }$, which is a parameter, the adversarial movement and activation schedule is limited by the following connectivity property:

Definition 2. Given a Mobile Ad-hoc Network, an instance of the Dissemination problem that starts at time $t_{1}$, and two integers $\alpha \geq 0$ and $\beta \geq 0$, the network is $(\alpha, \beta)$ connected if, for every time slot $t \geq t_{1}$ at which the problem has not yet been solved, there is a time slot $t^{\prime}$ such that the following conditions hold:

- the intersection of time intervals $[t, t+\alpha)$ and $\left[t^{\prime}, t^{\prime}+\beta\right)$ is not empty, and

- there is a pair of nodes $p, p^{\prime}$, such that at $t^{\prime} p$ is informed and $p^{\prime}$ is uncovered, and they are active and neighbors the whole time interval $\left[t^{\prime}, t^{\prime}+\beta\right)$.

It is of the utmost importance to notice that $(\alpha, \beta)$-connectivity is a characterization that applies to any model of dynamic network, given that for any mobility and activation schedule, and any pair of nodes, there is a minimum time they are connected (even if that time is 0) and a maximum time they are disconnected (even if that time is very large). Thus, any dynamic network model used to study the Dissemination problem has its own $\alpha$ and $\beta$ values.

Due to the same argument, $(\alpha, \beta)$-connectivity does not guarantee by itself that the network is epidemic (i.e. that the information is eventually disseminated); instead, an 
$(\alpha, \beta)$-connected network is only potentially epidemic. Consider for instance the source node. Thanks to the $(\alpha, \beta)$-connectivity, at most every $\alpha$ slots, the source $s$ is connected to other nodes of the network for at least $\beta$ time slots. But, we have progress only if the protocol to solve Dissemination is able to use the $\beta$ slots of connectivity to cover some uncovered node. As a consequence of the above discussed, impossibility results only restrict $\beta$, whereas $\alpha$ only constrains the running time, as it is shown in this paper.

\subsection{Protocols for Dissemination}

We consider distributed deterministic protocols, i.e., we assume that each node in the network is preloaded with its own and possibly different deterministic algorithm that defines a schedule of transmissions for it. Even if a transmission is scheduled for a given node at a given time, that node will not transmit if it is uninformed.

Following the literature on various communication primitives [16,17], a protocol is called oblivious if, at each node, the algorithm's decision on whether or not to schedule a transmission at a given time slot depends only on the identifier of the node, and on the number of time slots that the node has been active. Whereas, if no restriction is put on the information that a node may use to decide its communication schedule, the protocol is called adaptive. Additionally, in this paper, we distinguish a third class of protocols that we call quasi-oblivious. In a quasi-oblivious protocol the sequence of scheduled transmissions of a node depends only on its ID and a global time. Quasioblivious protocols have sometimes been called oblivious, since the model assumed simultaneous activation, and hence a global time was readily available. However we prefer to make the difference explicit, as done in [21], because we found a drastic gap between this class and fully oblivious protocols.

\subsection{Previous Work}

A survey of the vast literature related to Dissemination is beyond the scope of this article. We overview in this section the most relevant previous work. Additionally, a review of relevant related work for static and dynamic networks beyond MANETs can be found in [11].

The Dissemination problem abstracts several common problems in Radio Networks. When some number $1 \leq k \leq n$ of active nodes hold an information that must be disseminated to all nodes in the network, the problem is called $k$-Selection [16] or Manyto-all [5]. If $k=1$ the problem is called Broadcast [2, 18], whereas if $k=n$ the problem is known as Gossiping [4,9]. Upper bounds for these problems in mobile networks may be used for Dissemination, and even those for static networks may apply if the movement of nodes does not preclude the algorithm from completing the task (e.g., round-robin). On the other hand, if only the subset of $k$ nodes have to receive the information, the problem is known as Multicast [5,13], and if only nodes initially located at a parametric distance from the source node must receive the information the problem is called Geocast [15], defined in Section 1.1.

Deterministic solutions for the problems above have been studied for MANETs. Their correctness rely on strong synchronization or stability assumptions. In [19], deterministic Broadcast in MANETs was studied under the assumption that nodes move 
in a one-dimensional grid knowing their position. Two deterministic Multicast protocols for MANETs are presented in $[14,20]$. The solutions provided require the network topology to globally stabilize for long enough periods to ensure delivery of messages, and they assume a fixed number of nodes arranged in some logical or physical structure. Leaving aside channel contention, a lower bound of $\Omega(n)$ rounds of communication was proved in [23] for Broadcast in MANETs, even if nodes are allowed to move only in a two-dimensional grid, improving over the $\Omega(D \log n)$ bound of [3], where $D$ is the diameter of the network. This bound was improved to $\Omega(n \log n)$ in [7] without using the movement of nodes, but the diameter of the network in the latter is linear. Recently, deterministic solutions for Geocast were proposed in [1] for a one-dimensional setting and in [10] for the plane. In the latter work, the authors concentrate in the structure of the Geocast problem itself, leaving aside communication issues such as the contention for the communication channel.

\subsection{Our Results}

The main contribution of this work concerns negative results. Contrasting the lower bounds obtained with upper bounds derived by careful combination of previous techniques, interesting complexity gaps among protocol-classes are observed.

For a model where nodes may fail, there is no global clock, and nodes may be activated at different times, we show in Theorem 6 that any oblivious protocol takes, in the worst case, $\Omega\left(\left(\alpha+n^{2} / \ln n\right) n\right)$ steps to solve the Geocast problem if $v_{\max }>$ $\pi r / 6(\alpha+\lfloor(n / 3)(n / 3-1) / \ln (n / 3(n / 3-1))\rfloor-2)$. Given the upper bound of $n(\alpha+$ $4 n(n-1) \ln (2 n))$ for Dissemination established in Theorem 8 by means of an oblivious deterministic protocol based on Primed Selection [12], this lower bound is tight up to a poly-logarithmic factor.

Moreover, for the same model, Theorem 5 shows that, even if nodes are activated simultaneously and do not fail, and an adaptive protocol is used, any Geocast protocol takes, in the worst case, $\Omega(n(\alpha+n))$ if $v_{\max }>\pi r /(3(2 \alpha+n-4))$. This result should be contrasted with the quasi-oblivious protocol based on Round-Robin that solves Dissemination in at most $n(\alpha+n)$ steps as established in Theorem 7 .

The latter results are asymptotically tight and show that full adaptiveness does not help with respect to quasi-obliviousness. The first lower bound and the last upper bound, show an asymptotic separation almost linear between oblivious and quasi-oblivious protocols. In a more restrictive model, where nodes are activated simultaneously, there exists an oblivious protocol (e.g. Round Robin) that solves Dissemination in at most $n(\alpha+n)$ steps. Hence, the lower bound proved in Theorem 6 shows the additional cost of obliviousness when nodes are not simultaneously activated. This gap is what we call the profit of global synchrony, since it represents the gain the network obtains from global synchrony with respect to not having it. Moreover, the quasi-oblivious protocol derived shows that for the Dissemination problem, the simultaneous activation performance can be achieved by distributing the time elapsed since the source started the dissemination. For a discussion of the importance of node-activation schedule in distributed computing refer to [11].

Additionally, it is shown in Theorem 1 that no protocol can solve the Geocast problem (and hence Dissemination) in all $(\alpha, \beta)$-connected networks unless $\beta \geq n-1$. 
Interestingly, it is shown in Theorem 2 that this bound becomes $\beta>\lfloor(n-1)(n-$ $3) / 4 \ln ((n-1)(n-3) / 4)\rfloor$ if the protocol is oblivious. Comparing these bounds with the requirements of the protocols presented above, the quasi-oblivious protocol required $\beta \geq n$, which is almost optimal, while the oblivious protocol required $\beta \in \Omega\left(n^{2} \log n\right)$, which is only a polylogarithmic factor larger than the lower bound. These results also expose another aspect of the profit of global synchrony mentioned before: while $\beta=n$ is enough for quasi-oblivious protocols to solve Dissemination, oblivious protocols require a value of $\beta$ almost a linear factor larger.

Finally, for an arbitrary small bound on node speed, we show in Theorem 3 the existence of an $(\alpha, \beta)$-connected network where Geocast takes at least $\alpha(n-1)$ steps, even using randomization; and the existence of an $(\alpha, \beta)$-connected network where any deterministic protocol that transmits periodically takes at least $n(n-1) / 2$ steps, even if nodes do not move, in Theorem 4.

\subsection{Paper Organization}

The rest of the paper is organized as follows. In Section 2 we introduce some technical lemmas that will be used to prove our main results; in Section 3 we prove the lower bounds on link stability and on the time complexity to solve the Dissemination problem with respect to some important aspects of the system (e.g. speed of movement of nodes and their activation schedule) and of the protocols (e.g., obliviousness versus adaptiveness). We finally present the corresponding upper bounds in Section 4.

\section{Auxiliary Lemmas}

The following lemmas will be used throughout the analysis. A straightforward consequence of the pigeonhole principle is established in the following lemma.

Lemma 1. For any time step $t$ of the execution of a Dissemination protocol, where a subset $V^{\prime}$ of $k$ informed nodes do not fail during the interval $[t, t+k-2]$, there exists some node $v \in V^{\prime}$ such that $v$ does not transmit uniquely among the nodes in $V^{\prime}$ during the interval $[t, t+k-2]$.

In the following lemma, we show the existence of an activation schedule such that, for any oblivious deterministic protocol, within any subset of at least 3 nodes, there is one that does not have a unique transmission scheduled within a period roughly quadratic in the size of the subset. The proof, based on the probabilistic method, is omitted for brevity and can be found in [11].

Lemma 2. For any deterministic oblivious protocol that solves Dissemination in a MANET of $n$ nodes, where nodes are activated possibly at different times, and for any subset of $k$ nodes, $k \geq 3$, there exists a node-activation schedule such that, for any time slot $t$ and letting $m=\lfloor k(k-1) / \ln (k(k-1))\rfloor$, each of the $k$ nodes is activated during the interval $[t-m+1, t]$, and there is one of the $k$ nodes that is not scheduled to transmit uniquely among those $k$ nodes during the interval $[t, t+m-1]$. 


\section{Solvability of the Dissemination Problem}

If there is at least one node in $V_{\mathcal{P}}-\{s\}$ at least one time slot is needed to solve Dissemination, since the source node has to transmit at least once to pass the information. Furthermore, if all nodes in $V_{\mathcal{P}}$ are neighbors of $s$, one time slot may also be enough if the source node transmits before neighboring nodes are able to move out of its range. On the other hand, if the latter is not possible, more than one time slot may be needed. Let us consider the Geocast problem. Given that the specific technological details of the radio communication devices used determine the minimum running time when the eccentricity is $d \leq r$, all efficiency lower bounds are shown for $d>r$ unless otherwise stated.

\subsection{Link Stability Lower Bounds}

The following theorem shows a lower bound on the value of $\beta$ for the Geocast problem.

Theorem 1. For any $V_{\max }>0, d>r, \alpha>0$, and any deterministic Geocast protocol $\Pi$, if $\beta<n-1$, there exists an $(\alpha, \beta)$-connected MANET of n nodes such that $\Pi$ does not terminate, even if all nodes are activated simultaneously and do not fail.

Proof. Consider three sets of nodes $A, B$, and $C$ deployed in the plane, each set deployed in an area of size $\varepsilon$ arbitrarily small, such that $0<\varepsilon<r$ and $d \geq r+\varepsilon$. The invariant in this configuration is that nodes in each set form a clique, every node in $A$ is placed within distance $r$ from every node in $B$, every node in $B$ is placed at most at distance $\varepsilon$ from every node in $C$, and every node in $A$ is placed at some distance $r<\delta \leq r+\varepsilon$ from every node in $C$. At the beginning of the first time slot, the adversary places $n-1$ nodes, including the source node $s$, in the set $C$, the remaining node $x$ in set $A$, and activates all nodes. The set $B$ is initially empty. Given that $d \geq r+\varepsilon, x$ must become informed to solve the problem. Also, $\varepsilon$ is set appropriately so that a node can move $\varepsilon$ distance in one time slot without exceeding $V_{\max }$.

For any protocol $\Pi$ for Geocast, let $t$ be the first time slot where the source node is the only node to transmit in the set $C$. Adversarially, let $t$ be the first time slot when the source is informed. Thus, $(\alpha, \beta)$-connectivity is preserved up to time slot $t$ for any $\alpha$. At time slot $t$, all nodes placed in $C$ are informed.

After time slot $t$, the adversary moves the nodes as follows. Given that the problem was not solved yet and nodes in $C$ do not fail, according to Lemma 1 , there exists a node $y \in C$ that does not transmit uniquely among the nodes in $C$ during the interval $[t+1, t+n-2]$. Given that $\Pi$ is a deterministic protocol, and the adversary knows the protocol and defines the movement of all nodes, the adversary knows which is the node $y$.

Assume, for the sake of contradiction, that $\beta \leq n-2$. Then, the adversary places $y$ in $B$ for all time slots in the interval $[t+1, t+\beta]$. Additionally, for each time slot $t^{\prime} \in[t+1, t+\beta]$ where $y$ transmits, the adversary moves to $B$ some node $z \in C$ that transmits at $t^{\prime}$ to produce a collision. At the end of each time slot $t^{\prime}$ the adversary moves $z$ back to $C$. Such a node $z$ exists since $y$ does not transmit uniquely during the interval $[t+1, t+n-2]$ and $n-2 \geq \beta$. At the end of time slot $t+\beta$, the adversary moves $y$ back 
to $C$ and the above argument can be repeated forever preserving the $(\alpha, \beta)$-connectivity and precluding $\Pi$ from solving the problem. Therefore, $\beta$ must be at least $n-1$.

Building upon the argument used in the previous theorem, but additionally exploiting the adversarial node activation, the following theorem shows a lower bound on the value of $\beta$ for the Geocast problem if the protocol used is oblivious. The idea of the proof is to split evenly the nodes of set $C$ in the proof of Theorem 1 in two groups, so that alternately the nodes in one group are activated while the nodes in the other group produce collisions. The details are omitted for brevity and can be found in [11].

Theorem 2. For any $V_{\max }>0, d>r, n \geq 8, \alpha>0$, and any deterministic oblivious protocol for Geocast $\Pi$, if $\beta \leq m=\lfloor(n-1)(n-3) / 4 \ln ((n-1)(n-3) / 4)\rfloor$, there exists an $(\alpha, \beta)$-connected MANET of $n$ nodes such that $\Pi$ does not terminate.

\subsection{Time Complexity Lower Bounds versus Speed, Activation and Obliviousness}

Exploiting the maximum time $\alpha$ that a partition can be disconnected, a lower bound on the time efficiency of any protocol for Geocast, even regardless of the use of randomization and even for arbitrarily slow node-movement, can be proved. The following theorem establishes that bound. The proof is omitted for brevity and can be found in [11].

Theorem 3. For any $V_{\max }>0, d>r, \alpha>0$, and $\beta>0$, there exists an $(\alpha, \beta)$ connected MANET of $n$ nodes, for which any Geocast protocol takes at least $\alpha(n-1)$ time slots, even if all nodes are activated simultaneously and do not fail.

The linear lower bound for Geocast proved in Theorem 3 was shown exploiting the maximum time of disconnection between partitions. Exploiting the adversarial schedule of node activation, even if nodes do not move nor fail, the same bound can be simply proved for arbitrary Geocast protocols, while a quadratic bound can be shown for the important class of equiperiodic protocols. The protocol definition and the theorem for the latter follows. The proof is omitted for brevity and can be found in [11].

Definition 3. A protocol of communication for a Radio Network is equiperiodic if for each node, the transmissions scheduled are such that the number of consecutive time steps without transmitting, call it $T-1$, is always the same. We say that $T$ is the period of transmission of such a node.

Theorem 4. For any $V_{\max } \geq 0, d>r, \alpha>0, \beta>0$, and any deterministic equiperiodic Geocast protocol $\Pi$, there exists an $(\alpha, \beta)$-connected MANET of $n$ nodes, for which $\Pi$ takes at least $n(n-1) / 2$ time slots to solve the problem, even if nodes do not fail and do not move.

In Theorems 3 and 4 we showed lower bounds for Geocast for arbitrarily small values of $V_{\max }$. We now show that, by slightly constraining $V_{\max }$, a quadratic lower bound can also be shown for arbitrary deterministic protocols. 
Theorem 5. For any $V_{\max }>\pi r /(3(2 \alpha+n-4)), d>r, \alpha>0, \beta>0$, and any deterministic Geocast protocol $\Pi$, there exists an $(\alpha, \beta)$-connected MANET of n nodes, for which $\Pi$ takes $\Omega((\alpha+n) n)$ time slots to solve the problem, even if all nodes are activated simultaneously and do not fail.

Proof. The following adversarial configuration and movement of nodes shows the claimed lower bound. Consider six sets of nodes $A, A^{\prime}, B, B^{\prime}, C$, and $C^{\prime}$, each deployed in an area of size $\varepsilon$ arbitrarily small, such that $0<\varepsilon<r$ and $d \geq r+\varepsilon$, and four points, $x$, $y, x^{\prime}$, and $y^{\prime}$ placed in the configuration depicted in Figure 1(a).

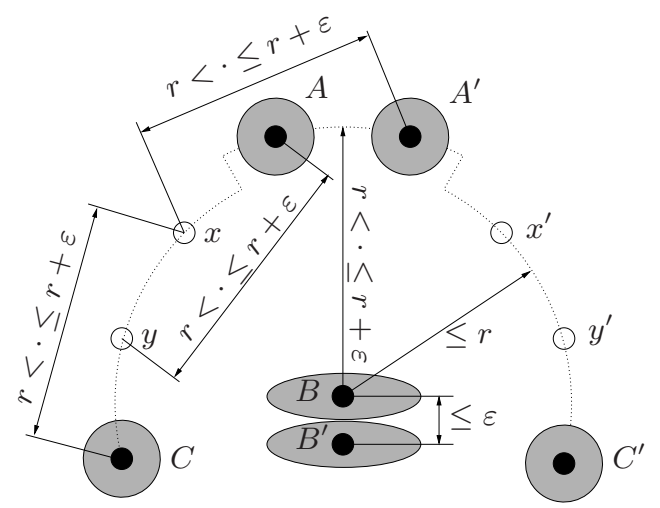

(a) Distances invariant.

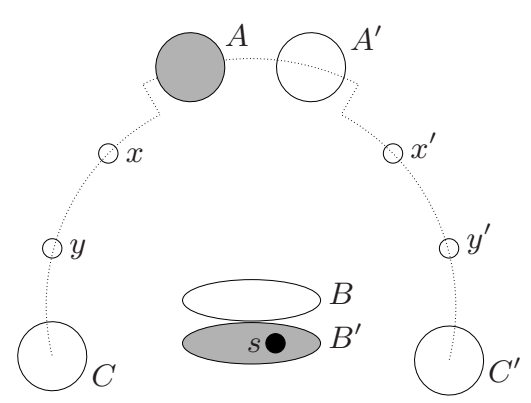

(b) Initial configuration.

Fig. 1. Illustration of Theorem 5. A small empty circle depicts a point in the plane. A small black circle depicts a node. A big empty area depicts an empty set. A big shaded area depicts a non-empty set.

The invariant in these sets is that nodes in each set form a clique; each node in $A^{\prime}$ is placed at some distance $>r$ and $\leq r+\varepsilon$ from the points $x, y^{\prime}$, and each node in $B$; each node in $A$ is placed at some distance $>r$ and $\leq r+\varepsilon$ from the points $x^{\prime}, y$, and each node in $B$; each node in $B$ is placed within distance $r$ of points $x, y, x^{\prime}$, and $y^{\prime}$, and each node in $C$ and $C^{\prime}$; each node in $C$ is placed at some distance $>r$ and $\leq r+\varepsilon$ from the point $x$; each node in $C^{\prime}$ is placed at some distance $>r$ and $\leq r+\varepsilon$ from the point $x^{\prime}$; and each node in $B^{\prime}$ is placed within distance $\varepsilon$ of each node in $B$ and within distance $r$ of each node in $C$ and $C^{\prime}{ }^{6}$

At the beginning of the first time slot, the adversary places $n / 2$ nodes, including the source node $s$, in set $B^{\prime}$, the remaining $n / 2$ nodes in the set $A$, and starts up all nodes. (For clarity, assume that $n$ is even.) All the other sets are initially empty. (See Figure 1(b).)Given that $d \geq r+\varepsilon$, all nodes must be covered to solve the problem. Also, $\varepsilon$ is set appropriately so that a node can be moved $\varepsilon$ distance in one time slot without exceeding $V_{\max }$, and so that a node can be moved from set $A$ to point $x$ through

\footnotetext{
${ }^{6}$ During some periods of time a given set could be empty, we mean that $x$ is separated (within) that distance from any point in the area designated to the set $X$
} 
the curved parts of the dotted line (see Figure 1(a)), of length less than $\pi(r+\varepsilon) / 6$, in $\alpha+n / 2-2$ time slots without exceeding $V_{\max }$. (To see why the length bound is that, it is useful to notice that the distance between each pair of singular points along each of the circular dotted lines is upper bounded by $(r+\varepsilon) / 2$.)

Let $t$ be the first time slot when the source is scheduled to transmit. Adversarially, let $t$ be the first time slot when the source is informed. Nodes stay in the positions described until $t$ and, consequently, all the other $n / 2-1$ nodes in set $B^{\prime}$ receive it. Starting at time slot $t+1$, the adversary moves the nodes so that only one new node every $\alpha+n / 2$ steps becomes informed. First we give the intuition of the movements and later the details. (See Figure 1(b).)Nodes that are not in $B$ or $B^{\prime}$ are moved following the dotted lines. Some of the nodes in $B^{\prime}$ are moved back and forth to $B$. Nodes initially in $A$ are moved clockwise to $A^{\prime}$, except for one of them, say $u$, which is moved simultaneously counterclockwise to the point $x$. Upon reaching $A^{\prime}$ nodes are moved counter-clockwise back to $A$, except for one of them, say $v$, which is moved simultaneously clockwise to the point $x^{\prime}$, while the node $u$ is also moved simultaneously to the point $y$. Upon reaching $A$, the remaining nodes repeat the procedure while $u$ keeps moving towards $C$ and $v$ keeps moving towards $C^{\prime}$ through $y^{\prime}$ respectively. Nodes initially in $A$ are moved in the above alternating fashion, one to $C$ and the next one to $C^{\prime}$, until all nodes become informed. Movements are produced so that $(\alpha, \beta)$-connectivity is preserved. The details follow.

The movement of each node $u$ moved from $A$ to $C$ is carried out in three phases of at least $\alpha+n / 2-2$ time slots each as follows. (As explained below, some nodes initially in $A$ will be moved instead to $C^{\prime}$, but the movement is symmetric. For clarity, we only describe the whole three phases for one node. The movement is illustrated in Figure 4 in [11], which is omitted here for brevity.)

Phase 1 During the first $\alpha-2$ time slots, $u$ is moved counterclockwise from $A$ towards the point $x$ maintaining a distance $>r$ and $\leq r+\varepsilon$ with respect to every node in $B$. In the $(\alpha-1)$-th time slot of this phase, $u$ is moved within distance $r$ of every node in set $B$ preserving $(\alpha, \beta)$-connectivity. Nodes in $B^{\prime}$ stay static during this interval. Given that only nodes in $B^{\prime}$ are informed and the distance between them and $u$ is bigger than $r, u$ does not become covered during this interval.

During the following $n / 2-1$ time slots of the first phase, the counterclockwise movement of node $u$ towards the point $x$ continues, but now maintaining a distance at most $r$ with respect to every node in $B$. In the last time slot of the second phase, $u$ is moved to point $x$. During this interval, nodes in $B^{\prime}$ are moved back and forth to $B$ as described in Theorem 1 to guarantee that $u$ does not become covered before reaching point $x$. Upon reaching point $x, u$ and all the other nodes in the network not in $B$ or $B^{\prime}$ remain static. Phase 1 ends the time slot before $u$ becomes covered. Simultaneously, along the first $\alpha+n / 2-2$ time steps of this phase, the remaining nodes initially in $A$ are moved clockwise to $A^{\prime}$. Then, even if $u$ becomes informed immediately upon reaching point $x, u$ cannot inform nodes in $A^{\prime}$ because they are separated by a distance $>r$.

Phase 2 During this phase, $u$ is moved counterclockwise towards point $y$ maintaining a distance at most $r$ with respect to every node in $B$ and $B^{\prime}$. Simultaneously, nodes that were in $A^{\prime}$ at the end of the second phase are moved counterclockwise to $A$ except for one node $v$ that moves in its own first phase to $x^{\prime}$. 
Nodes moving from $A^{\prime}$ to $A$ maintain a distance $>r$ with respect to $u$. Thus, even if $u$ becomes covered the information cannot be passed to the former. At the end of this phase $v$ is placed in point $x^{\prime}$. Thus, even if $v$ becomes covered in the first step of its second phase, $v$ cannot inform nodes in $A$ because they are separated by a distance $>r$.

Phase 3 During this phase, $u$ is moved counterclockwise towards set $C$ maintaining a distance at most $r$ with respect to every node in $B$ and $B^{\prime}$. Simultaneously, nodes that were in $A$ at the end of the second phase are moved clockwise to $A^{\prime}$ except for one node $w$ that moves in its own first phase to $x$. Also simultaneously, $v$ continues its movement towards set $C^{\prime}$ in its own second phase.

Nodes moving from $A$ to $A^{\prime}$ maintain a distance $>r$ with respect to $v$. Thus, even if $v$ becomes covered the information cannot be passed to the former. Also, nodes $u$ and $w$ are moved maintaining a distance $>r$ between them. Thus, $u$ cannot inform $w$. At the end of this phase $u$ has reached set $C, v$ is placed in point $y^{\prime}$, and $w$ is placed in point $x$. Thus, even if $w$ becomes covered in the first step of its second phase, $w$ cannot inform nodes in $A$ because they are separated by a distance $>r$. Upon completing the third phase, $u$ stays static in $C$ forever so that $(\alpha, \beta)$ connectivity is preserved.

The three-phase movement detailed above is produced for each node initially in $A$, overlapping the phases as described, until all nodes have became covered. Given that when a node $u$ reaches the point $x$, its phase 1 is stretched until the time step before $u$ becomes covered by a node $v$ in $B$ and all other nodes remain static, the next node $w$ that will be moved from $A^{\prime}$ to $x^{\prime}$ does not become covered by $v$, because $w$ stays in $A^{\prime}$ until $u$ becomes covered. In each phase of at least $\alpha+n / 2-2$ time slots every node is moved a distance at most $\pi(r+\varepsilon) / 6+\varepsilon$. Thus, setting $\varepsilon$ appropriately, the adversarial movement described does not violate $V_{\max }$. Given that $n / 2$ nodes initially in $A$ are covered one by one, each at least within $\alpha+n / 2-2$ time slots after the previous one, the overall running time is lower bounded as claimed, even if $t=1$.

The quadratic lower bound shown in Theorem 5 holds for any deterministic protocol, even if it is adaptive. Building upon the argument used in that theorem, but additionally exploiting the adversarial node activation, the following theorem shows a roughly cubic lower bound for oblivious protocols, even relaxing the constraint on $V_{\max }$. The proof is omitted for brevity, the details can be found in [11].

Theorem 6. For any $n \geq 9, d>r, \alpha>0, \beta>0, V_{\max }>\pi r / 6(\alpha+\lfloor(n / 3)(n / 3-$ $1) / \ln (n / 3(n / 3-1))\rfloor-2)$, and any oblivious deterministic Geocast protocol $\Pi$, there exists an $(\alpha, \beta)$-connected MANET of $n$ nodes, for which $\Pi$ takes $\Omega\left(\left(\alpha+n^{2} / \ln n\right) n\right)$ time slots to solve the problem.

\section{Upper Bounds}

Solving the Dissemination problem under arbitrary node-activation schedule and nodemovement is not a trivial task. To the best of our knowledge, deterministic protocols for such scenarios were not studied before, not even for potentially epidemic networks such 
as an $(\alpha, \beta)$-connected MANET, and not even for specific instances of Dissemination. In this section, a quasi-oblivious protocol and an oblivious one that solve Dissemination, both based on known algorithms particularly suited for our setting, are described and their time efficiency proved. The first bound is asymptotically tight with respect to the more powerful class of adaptive protocols.

A Quasi-Oblivious Protocol. The idea behind the protocol is to augment the wellknown Round-Robin protocol with the synchronization of the clock of each node with the time elapsed since the dissemination started, which we call the global time. This is done by embedding a counter $\tau$, corresponding to the global time, in the messages exchanged to disseminate the information $I$. Given that the schedule of transmissions of a node depends only on its ID and the global time, the protocol is quasi-oblivious. More details about the algorithm can be found in [11].

It can be proved that this quasi-oblivious algorithm solves Dissemination for arbitrary values of $V_{\max }$ in at most $n(\alpha+n)$ time steps. The details are omitted for brevity and can be found in [11]. Formally,

Theorem 7. Given an $(\alpha, \beta)$-connected MANET where $\beta \geq n$, there exists a quasioblivious deterministic protocol that solves Dissemination for arbitrary values of $V_{\max }$ in at most $n(\alpha+n)$ time steps.

Recall that $\beta \geq n-1$ is required for the problem to be solvable as shown in Theorem 1. This upper bound is asymptotically tight with respect to the lower bound for general deterministic Geocast protocols when $V_{\max }>\pi r /(3(2 \alpha+n-4))$ shown in Theorem 5. Thus, we can conclude that having extra information in this case does not help.

An Oblivious Protocol. We finally describe how to implement an oblivious protocol for Dissemination, based on Primed Selection, a protocol presented in [12] for the related problem of Recurrent Communication. Given that in this protocol the schedule of transmissions of a node depends only on its ID, the protocol is oblivious. This upper bound is only a poly-logarithmic factor away from the lower bound shown in Theorem 6 .

In order to implement Primed Selection, one of $n$ prime numbers is stored in advance in each node's memory, so that each node holds a different prime number. Let $p_{\ell}$ denote the $\ell$-th prime number. We set the smallest prime number used to be $p_{n}$, which is at least $n$, because Primed Selection requires the smallest prime number to be at least the maximum number of neighbors of any node, which in our model is unknown. The algorithm is simple to describe, upon receiving the information, each node with assigned prime number $p_{i}$ transmits with period $p_{i}$.

It was shown in [12] that, for any Radio Network formed by a set $V$ of nodes running Primed Selection, for any time slot $t$, and for any node $i$ such that the number of nodes neighboring $i$ is $k-1,1<k<n, i$ receives a transmission without collision from each of its neighbors within at $\operatorname{most}_{k} \max _{j \in V} p_{j}$ steps after $t$. Given that in our setting the biggest prime number used is $p_{2 n-1}$, that $p_{x}<x(\ln x+\ln \ln x)$ for any $x \geq 6$ as shown in [25], and that due to mobility all nodes may get close to $i$ in the worst case, we have that $k \max _{j \in V} p_{j}<n(2 n-1)(\ln (2 n-1)+\ln \ln (2 n-1))$, for $n \geq 4$. Which 
is in turn less than $4 n(n-1) \ln (2 n)$ for $n \geq 3$. Hence, given that in the worst case all nodes must be covered at least one at a time and that the network is $(\alpha, \beta)$-connected, the overall running time is less than $n(\alpha+4 n(n-1) \ln (2 n))$. We formalize this bound in the following theorem. Recall that $\beta>\lfloor(n-1)(n-3) / 4 \ln ((n-1)(n-3) / 4)\rfloor$ is required for the problem to be solvable when $n \geq 8$ as shown in Theorem 2 .

Theorem 8. Given an $(\alpha, \beta)$-connected MANET, where $\beta \geq n(2 n-1)(\ln (2 n-1)+$ $\ln \ln (2 n-1))$ and $n \geq 4$, there exists an oblivious deterministic protocol that solves Dissemination for arbitrary values of $V_{\max }$ in at most $n(\alpha+4 n(n-1) \ln (2 n))$ time steps.

\section{References}

1. R. Baldoni, K. Ioannidou, and A. Milani. Mobility versus the cost of geocasting in mobile ad-hoc networks. In Proc. 21st Ann. EATCS International Symposium on Distributed Computing, volume 4731 of Lecture Notes in Computer Science, pages 48-62. Springer-Verlag, Berlin, 2007.

2. R. Bar-Yehuda, O. Goldreich, and A. Itai. On the time-complexity of broadcast in multi-hop radio networks: An exponential gap between determinism and randomization. Journal of Computer and System Sciences, 45:104-126, 1992.

3. D. Bruschi and M. Del Pinto. Lower bounds for the broadcast problem in mobile radio networks. Distributed Computing, 10(3):129-135, 1997.

4. B. Chlebus, L. Gạsieniec, A. Lingas, and A. Pagourtzis. Oblivious gossiping in ad-hoc radio networks. In Proc. of 5th Intl. Workshop on Discrete Algorithms and Methods for Mobile Computing and Communications, pages 44-51, 2001.

5. B. S. Chlebus, D. R. Kowalski, and T. Radzik. On many-to-many communication in packet radio networks. In Proceedings of the 10th International Conference on Principles of Distributed Systems, pages 258-272, 2006.

6. A.E.F. Clementi, F. Pasquale, A. Monti, and R. Silvestri. Communication in dynamic radio networks. In Proc. 26th Ann. ACM Symp. on Principles of Distributed Computing, pages 205-214, 2007.

7. A. Dessmark and A. Pelc. Broadcasting in geometric radio networks. Journal of Discrete Algorithms, 5:187-201, 2007.

8. K. Fall. A delay-tolerant network architecture for challenged internets. In Proceedings of the 2003 conference on Applications, technologies, architectures, and protocols for computer communications (SIGCOMM), pages 27-34, 2003.

9. M. Farach-Colton and M. A. Mosteiro. Sensor network gossiping or how to break the broadcast lower bound. In Proc. of the 18th Intl. Symp. on Algorithms and Computation, volume 4835 of Lecture Notes in Computer Science, pages 232-243. Springer-Verlag, Berlin, 2007.

10. A. Fernández Anta and A. Milani. Bounds for deterministic reliable geocast in mobile ad-hoc networks. In Proceedings of the 12th International Conference on Principles of Distributed Systems, pages 164-183, 2008.

11. A. Fernández Anta, A. Milani, M. A. Mosteiro, and S. Zaks. Opportunistic information dissemination in mobile ad-hoc networks: The profit of global synchrony. Technical Report RoSaC-2010-1, GSyC, Universidad Rey Juan Carlos, 2010.

12. A. Fernández Anta, Miguel A. Mosteiro, and Christopher Thraves. Deterministic communication in the weak sensor model. In Proceedings of the 11th International Conference on Principles of Distributed Systems, pages 119-131, 2007. 
13. L. Gasieniec, E. Kranakis, A. Pelc, and Q. Xin. Deterministic $\mathrm{m} 2 \mathrm{~m}$ multicast in radio networks. In Proc. of 31 st Intl. Colloquium on Automata Languages and Programming, pages 670-682, 2004.

14. S.K.S. Gupta and P.K. Srimani. An adaptive protocol for reliable multicast in mobile multihop radio networks. In Proceedings of the 2nd IEEE Workshop on Mobile Computing Systems and Applications, 1999.

15. X. Jinag and T. Camp. A review of geocasting protocols for a mobile ad hoc network. In Proceedings of Grace Hopper Celebration, 2002.

16. D. R. Kowalski. On selection problem in radio networks. In Proc. 24th Ann. ACM Symp. on Principles of Distributed Computing, pages 158-166, 2005.

17. D. R. Kowalski and A. Pelc. Time complexity of radio broadcasting: Adaptiveness vs. obliviousness and randomization vs. determinism. Theoretical Computer Science, 333:355-371, 2005.

18. E. Kushilevitz and Y. Mansour. An $\Omega(D \log (N / D))$ lower bound for broadcast in radio networks. SIAM Journal on Computing, 27(3):702-712, 1998.

19. M. Mohsin, D. Cavin, Y. Sasson, R. Prakash, and A. Schiper. Reliable broadcast in wireless mobile ad hoc networks. In Proceedings of the 39th Hawaii International Conference on System Sciences, page 233, 2006.

20. E. Pagani and G.P. Rossi. Reliable broadcast in mobile multihop packet networks. In Proc. of the 3rd ACM Ann. Intl. Conference on Mobile Computing and Networking, pages 34-42, 1997.

21. D. Peleg and Tomasz Radzik. Time-efficient broadcast in radio networks. In A. M. C. A. Koster and Xavier Muñoz, editors, Graphs and Algorithms in Communication Networks, chapter 12, pages 315-339. Springer-Verlag, 2009.

22. L. Pelusi, A. Passarella, and M. Conti. Opportunistic networking: data forwarding in disconnected mobile ad hoc networks. Communications Magazine, IEEE, 44(11):134-141, 2006.

23. R. Prakash, A. Schiper, M. Mohsin, D. Cavin, and Y. Sasson. A lower bound for broadcasting in mobile ad hoc networks. Technical report, Ecole Polytechnique Federale de Lausanne, 2004.

24. L. G. Roberts. Aloha packet system with and without slots and capture. Computer Communication Review, 5(2):28-42, 1975.

25. J. B. Rosser and L. Schoenfeld. Approximate formulas for some functions of prime numbers. Illinois Journal of Mathematics, 6(1):64-94, 1962. 